\title{
Tumor-specific immunity and antiangiogenesis generated by a DNA vaccine encoding calreticulin linked to a tumor antigen
}

\author{
Wen-Fang Cheng, ${ }^{1,2}$ Chien-Fu Hung, ${ }^{1}$ Chee-Yin Chai, ${ }^{1}$ Keng-Fu Hsu, ${ }^{1}$ Liangmei He, ${ }^{1}$ \\ Morris Ling, ${ }^{1}$ and T.-C. Wu $\mathrm{W}^{1,3,4,5}$
}

${ }^{1}$ Departments of Pathology, Johns Hopkins Medical Institutions, Baltimore, Maryland, USA
2Department of Obstetrics and Gynecology, National Taiwan University Hospital, Taipei, Taiwan
${ }^{3}$ Obstetrics and Gynecology,
${ }^{4}$ Molecular Microbiology and Immunology, and
${ }^{5}$ Oncology, Johns Hopkins Medical Institutions, Baltimore, Maryland, USA

Address correspondence to: T.-C. Wu, Department of Pathology, the Johns Hopkins University School of Medicine, Ross 659, 720 Rutland Avenue, Baltimore, Maryland 21205, USA. Phone: (410) 614-3899; Fax: (410) 614-3548; E-mail: wutc@jhmi.edu.

Wen-Fang Cheng and Chien-Fu Hung contributed equally to this work.

Received for publication January 25, 2001, and accepted in revised form July 26, 2001.

\begin{abstract}
Antigen-specific cancer immunotherapy and antiangiogenesis have emerged as two attractive strategies for cancer treatment. An innovative approach that combines both mechanisms will likely generate the most potent antitumor effect. We tested this approach using calreticulin (CRT), which has demonstrated the ability to enhance MHC class I presentation and exhibit an antiangiogenic effect. We explored the linkage of CRT to a model tumor antigen, human papilloma virus type-16 (HPV-16) $\mathrm{E} 7$, for the development of a DNA vaccine. We found that $\mathrm{C} 57 \mathrm{BL} / 6$ mice vaccinated intradermally with CRT/E7 DNA exhibited a dramatic increase in E7-specific $\mathrm{CD}^{+} \mathrm{T}$ cell precursors and an impressive antitumor effect against $\mathrm{E} 7$-expressing tumors compared with mice vaccinated with wild-type E7 DNA or CRT DNA. Vaccination of CD4/CD8 double-depleted C57BL/6 mice and immunocompromised (BALB/c $n u / n u)$ mice with CRT/E7 DNA or CRT DNA generated significant reduction of lung tumor nodules compared with wild-type E7 DNA, suggesting that antiangiogenesis may have contributed to the antitumor effect. Examination of microvessel density in lung tumor nodules and an in vivo angiogenesis assay further confirmed the antiangiogenic effect generated by CRT/E7 and CRT. Thus, cancer therapy using CRT linked to a tumor antigen holds promise for treating tumors by combining antigen-specific immunotherapy and antiangiogenesis.
\end{abstract}

J. Clin. Invest. 108:669-678 (2001). DOI:10.1172/JCI200112346.

\section{Introduction}

The ideal cancer treatment should be able to eradicate systemic tumors at multiple sites in the body while having the specificity to discriminate between neoplastic and nonneoplastic cells. In this regard, antigen-specific cancer immunotherapy and antiangiogenesis represent two attractive approaches for cancer treatment. Activation of antigen-specific $\mathrm{T}$ cell-mediated immune responses allows for killing of tumors associated with a specific antigen $(1,2)$ while inhibition of angiogenesis controls neoplastic growth by sequestering neoplastic cells from an adequate blood supply $(3,4)$. Therefore, an innovative approach that combines both mechanisms will likely generate the most potent antitumor effect.

The use of calreticulin (CRT) represents a feasible approach for enhancing tumor-specific $\mathrm{T}$ cell-mediated immune responses and generating an antiangiogenic effect. CRT is an abundant $46 \mathrm{kDa} \mathrm{Ca}^{2+}$-binding protein located in the endoplasmic reticulum (ER) (5). CRT is considered to be related to the family of heat shock proteins (HSPs) $(6,7)$. The protein has been shown to associate with peptides delivered into the ER by transporters associated with antigen processing (TAP-1 and TAP-2) (8) and with MHC class I- $\beta 2$ microglobulin molecules to aid in antigen presentation (9). Previous studies have shown that CRT can be complexed with peptides in vitro to elicit peptide-specific $\mathrm{CD}^{+} \mathrm{T}$ cell responses through exogenous administration (7). In addition, peptide-bound CRT purified from tumor extracts has been shown to elicit an antitumor effect specific to the source tumor (7). These data indicate that cancer vaccines employing CRT can potentially enhance tumor-specific immune responses.

Tumor growth and metastasis formation is dependent on the existence of an adequate blood supply. As neoplasms grow larger, adequate blood supply to the tumor tissue is often ensured by new vessel formation, a process termed angiogenesis. Therapeutic agents that target tumor vasculature may prevent or delay tumor growth and even promote tumor regression or dormancy $(4,10)$. Recently, CRT and its protein fragment (aa 1-180), vasostatin, have been reported to be endothelial cell inhibitors of tumor growth $(11,12)$. Therefore, cancer vaccines employing CRT may poten- 
tially generate antitumor effects through enhanced antitumor immune responses and antiangiogenic effects. We investigated the novel use of CRT chimerically linked to a model antigen (HPV-16 E7) for the treatment of tumors through the combination of antigen-specific immunotherapy and antiangiogenesis.

\section{Methods}

Plasmid DNA constructs and preparation. The generation of pcDNA3-E7, pcDNA3-green fluorescent protein (pcDNA3-GFP), and pcDNA3-E7/GFP has been described previously (13). For the generation of pcDNA3-CRT, CRT was first amplified with PCR using rabbit CRT cDNA as the template (14) and a set of primers, 5 '-CCGGTCTAGAATGCTGCTCCCTGTGCCGCT$3^{\prime}$ and $5^{\prime}$-CCGGGAATTCCAGCTCGTCCTTGGCCTGGC- ${ }^{\prime}$. There is more than $90 \%$ homology between rabbit, human, mouse, and rat CRT (14). The amplified product was then cloned into the $\mathrm{XbaI} / \mathrm{EcoRI}$ sites of pcDNA3 vector (Invitrogen Corp., Carlsbad, California, USA). For the generation of pcDNA3-CRT/E7, E7 was first amplified with a set of primers, $5^{\prime}$-ggggaattcatggagatacaccta- $3^{\prime}$ and $5^{\prime}$-ggtggatccttgagaacagatgg- $3^{\prime}$, and cloned into the EcoRI/BamHI sites of pcDNA3-CRT to generate pcDNA3-CRT/E7. To generate pcDNA3CRT/E7/GFP, the CRT DNA fragment was isolated from pcDNA3-CRT and cloned into Xbal/EcoRI sites of pcDNA3-E7/GFP. Plasmid constructs were confirmed by DNA sequencing.

Transfection of $293 D^{b}, K^{b}$ cells and confocal fluorescence microscopy. E7/GFP or CRT/E7/GFP DNA were transfected into $293 \mathrm{D}^{\mathrm{b}}, \mathrm{K}^{\mathrm{b}}$ cells, stained with mouse anticalnexin mAb (StressGen Biotechnologies Corp., Victoria, British Columbia, Canada) followed by Cy3-conjugated $\mathrm{F}\left(\mathrm{ab}^{\prime}\right)_{2}$ fragment goat anti-mouse IgG (Jackson ImmunoReseach Laboratories Inc., West Grove, Pennsylvania, USA), and examined under a confocal laser scanning microscope using a protocol described previously (15).

DNA vaccination. Preparation of DNA-coated gold particles and gene gun particle-mediated DNA vaccination were performed using a helium-driven gene gun (BioRad Laboratories Inc., Hercules, California, USA) according to a protocol described previously (16). Control plasmid, E7, CRT, or CRT/E7 DNA-coated gold particles were delivered to the shaved abdominal region of mice using a helium-driven gene gun (BioRad Laboratories Inc.) with a discharge pressure of 400 psi.

Intracellular cytokine staining and flow cytometry analysis. Mice were immunized with $2 \mu \mathrm{g}$ of the various DNA vaccines and received a booster with the same regimen 1 week later. Splenocytes were harvested 1 week after the last vaccination. Before intracellular cytokine staining, $3.5 \times 10^{5}$ pooled splenocytes from each vaccination group were incubated for 16 hours with either $1 \mu \mathrm{g} / \mathrm{ml}$ of E7 peptide (aa 49-57) containing an MHC class I epitope (17) for detecting E7-specific $\mathrm{CD}^{+} \mathrm{T}$ cell precursors or $10 \mu \mathrm{g} / \mathrm{ml}$ of E7 peptide (aa 30-67) containing an MHC class II epitope (18) for detecting E7-specific
$\mathrm{CD} 4^{+} \mathrm{T}$ cell precursors. Cell surface marker staining of CD8 or CD4 and intracellular cytokine staining for IFN- $\gamma$ and IL-4, as well as flow cytometry analysis, were performed using conditions described previously (16). ELISA. The presence of anti-HPV $16 \mathrm{E7} \mathrm{Ab}$ 's in the sera was characterized by a direct ELISA as described previously (19). Mice were immunized with $2 \mu \mathrm{g}$ of the various DNA vaccines and received a booster with the same regimen 1 week later. Sera were prepared from mice on day 14 after immunization. The ELISA plate was read with a standard ELISA reader at $450 \mathrm{~nm}$.

For the detection of CRT or CRT/E7 protein concentration in the sera of vaccinated mice, we performed an indirect ELISA using a protocol similar to one described previously (20). Briefly, mice were immunized with 16 $\mu \mathrm{g}$ of CRT or CRT/E7 DNA and received a booster with the same regimen 1 week later. Sera were collected 3, 7, 11,14 , and 18 days after the last immunization. Sera were then serially diluted in PBS, coated in a 96-microwell plate, and incubated at $4{ }^{\circ} \mathrm{C}$ overnight. The wells were then blocked with PBS containing $20 \%$ FBS. After washing with PBS containing $0.05 \%$ Tween-20, the plate was incubated with a 1:1,000 dilution of rabbit anti-CRT Ab (StressGen Biotechnologies Corp.) for 2 hours at $37^{\circ} \mathrm{C}$. The plate was further incubated with 1:1,000 dilution of a peroxidase-conjugated donkey anti-rabbit IgG Ab (Amersham Pharmacia Biotech, Piscataway, New Jersey, USA) at room temperature for 1 hour. The plate was washed, developed with 1-Step Turbo TMB-ELISA (Pierce Chemical Co., Rockford, Illinois, USA), and stopped with $1 \mathrm{M} \mathrm{H}_{2} \mathrm{SO}_{4}$. The concentration of CRT or CRT/E7 protein was determined by comparison to a standard curve of purified recombinant CRT protein from bacteria.

Cytotoxic T lymphocyte assay using transfected $293 D^{b}, K^{b}$ cells as target cells. Cytotoxic T lymphocyte (CTL) assays were performed by quantitative measurements of lactate dehydrogenase (LDH) using CytoTox96 nonradioactive cytotoxicity assay kits (Promega Corp., Madison, Wisconsin, USA) according to the manufacturer's protocol. Various DNA-transfected $293 \mathrm{D}^{\mathrm{b}}, \mathrm{K}^{\mathrm{b}}$ cells served as target cells while a $\mathrm{D}^{\mathrm{b}}$-restricted E7-specific $\mathrm{CD}^{+} \mathrm{T}$ cell line (21) was used as effector cells. Untransfected $293 \mathrm{D}^{\mathrm{b}}, \mathrm{K}^{\mathrm{b}}$ cells were used as a negative control. The $293 \mathrm{D}^{\mathrm{b}}, \mathrm{K}^{\mathrm{b}}$ cells were collected $40-44$ hours after transfection. CTL assays were performed with effector cells and target cells $\left(10^{4}\right.$ per well) mixed together at various effector/target (E/T) ratios, 1:1, 3:1, 9:1, and 27:1, in a final volume of $200 \mu \mathrm{l}$. After a 5 -hour incubation at $37^{\circ} \mathrm{C}, 50 \mu \mathrm{l}$ of the cultured media were collected to assess the amount of LDH in the cultured media. The percentage of lysis was calculated from the following equation: $100 \times(A-B) /(C-D)$, where $A$ is the reading of experimental-effector signal value, $B$ is the effector spontaneous background signal value, $C$ is maximum signal value from target cells, $D$ is the target spontaneous background signal value.

CTL assay using dendritic cells pulsed with lysates of transfected $293 D^{b}, K^{b}$ cells as target cells. CTL assays were per- 
formed using freshly isolated dendritic cells (DCs) pulsed with cell lysates as target cells and $\mathrm{D}^{\mathrm{b}}$-restricted E7-specific CD8 ${ }^{+} \mathrm{T}$ cells as effector cells using a protocol similar to one described previously (15). The protein concentration of lysates was determined using the BioRad protein assay (BioRad Laboratories Inc.) according to vendor's protocol, and the amount of E7 protein was checked using a standard ELISA (20). Cell lysates from E7 or CRT/E7 DNA-transfected $293 \mathrm{D}^{\mathrm{b}}, \mathrm{K}^{\mathrm{b}}$ cells were standardized for $\mathrm{E} 7$ protein concentration. DCs were prepared by pulsing with different concentrations of cell lysates of various DNA-transfected $293 \mathrm{D}^{\mathrm{b}}, \mathrm{K}^{\mathrm{b}}$ cells $(50 \mu \mathrm{g} / \mathrm{ml}, 10 \mu \mathrm{g} / \mathrm{ml}$, $2 \mu \mathrm{g} / \mathrm{ml}$, and $0.4 \mu \mathrm{g} / \mathrm{ml}$ ) in a final volume of $2 \mathrm{ml}$ for 16-20 hours. CTL assays were performed at a fixed $\mathrm{E} / \mathrm{T}$ ratio of 9:1 using $9 \times 10^{4} \mathrm{E} 7$-specific $\mathrm{T}$ cells mixed with $1 \times 10^{4}$ prepared DCs in a final volume of $200 \mu \mathrm{l}$. Cytolysis was determined by quantitative measurements of LDH as described earlier.

In vivo tumor protection experiments. For the tumor protection experiment, C57BL/6 mice (five per group) either received no vaccination or were immunized with $2 \mu \mathrm{g} /$ mouse of plasmid without insert, CRT, E7, CRT/E7, or CRT mixed with E7 (CRT + E7) DNA with a gene gun. One week later, mice were boosted with the same regimen as the first vaccination. One week after the last vaccination, mice were subcutaneously challenged with $5 \times 10^{4} \mathrm{TC}-1$ cells/mouse in the right leg. Mice were monitored for evidence of tumor growth by palpation and inspection twice a week until they were sacrificed at day 60 .

In vivo $\mathrm{Ab}$ depletion experiments. In vivo $\mathrm{Ab}$ depletions were performed as described previously (22). Briefly, C57BL/6 mice (five per group) were vaccinated with 2 $\mu \mathrm{g} / \mathrm{mouse}$ of CRT/E7 DNA with a gene gun, boosted 1 week later, and challenged with $5 \times 10^{4}$ cells/mouse TC-1 tumor cells. Depletion was started 1 week before tumor challenge. The mAb GK1.5 was used for CD4 depletion, $\mathrm{mAb} 2.43$ was used for $\mathrm{CD} 8$ depletion, and mAb PK136 was used for NK1.1 depletion. Depletion was terminated on day 40 after tumor challenge.

In vivo tumor treatment experiments. In vivo tumor treatment experiments were performed using a previously described lung hematogenous spread model (23). C57BL/6 mice or nude (BALB/c nu/nu) mice (five per group) were challenged with $10^{4}$ cells/mouse TC- 1 tumor cells via tail vein. Two days after tumor challenge, mice received $16 \mu \mathrm{g} /$ mouse of CRT DNA, E7 DNA, CRT mixed with E7 DNA, or CRT/E7 DNA by a gene gun, followed by a booster with the same regimen every 7 days for 3 weeks (a total of four shots, $64 \mu \mathrm{g}$ DNA). Mice receiving no vaccination were used as a negative control. Mice were sacrificed and lungs were explanted on day 28. The pulmonary tumor nodules in each mouse were evaluated and counted by experimenters blinded to sample identity.

We also performed an in vivo tumor treatment experiment in the various DNA-treated mice depleted of both $\mathrm{CD}^{+}$and $\mathrm{CD} 8^{+} \mathrm{T}$ cells using a protocol similar to one described previously (20). Double depletion was started 1 week before tumor challenge using $\mathrm{mAb}$ GK1.5 for CD4 depletion and mAb 2.43 for CD8 depletion. Depletion was continued until mice were sacrificed on day 28. Lung tumor nodules were counted as described above. The completeness of depletion was confirmed by flow cytometry analysis (22).

Immunobistochemical labeling for the quantitation of microvessel density. Labeling of intratumoral microvessels was performed with rat anti-mouse CD31 mAb (1:25 dilution; PharMingen), followed by biotinylated goat anti-rat $\mathrm{Ab}$ (1:100 dilution; Jackson ImmunoReseach Laboratories Inc.) and streptavidin-conjugated horseradish peroxidase (DAKO Corp., Carpinteria, California, USA) using a protocol previously described (24). The method for quantitating microvessel density (MVD) has also been described previously (25). Briefly, slides were prepared and examined at $\times 40$ and $\times 100$. In each section, the three most vascularized areas were chosen. Microvessel counts were obtained at $\times 200$ and the mean number in the three fields for each tumor was calculated and referred to as the MVD count. Large vessels with thick muscular walls and lumina greater than approximately eight blood cells were excluded from the count. We counted and compared MVD in tumors of similar size to minimize the influence of tumor size on the measurements (since smaller tumor nodules tend to have smaller MVD). All measurements were performed by a single pathologist blinded to sample identity.

In vivo angiogenesis assay using Matrigel. In vivo angiogenesis was assessed using the Matrigel plug assay with a protocol similar to that described previously $(26,27)$. Mice were immunized with $16 \mu \mathrm{g}$ of plasmid without insert, wild-type E7, CRT, or CRT/E7 DNA on day 0 and received a booster with the same regimen on day 7 . Matrigel (Becton Dickinson and Co., Franklin Lakes, New Jersey, USA) was mixed with heparin (final concentration of $50 \mathrm{U} / \mathrm{ml}$ ) and bFGF (final concentration of $10 \mathrm{ng} / \mathrm{ml}$ ) at $4^{\circ} \mathrm{C}$. A total of $0.5 \mathrm{ml} /$ mouse of this Matrigel mixture was injected subcutaneously into the abdominal midline of DNA-vaccinated mice on day 7. Naive mice injected with Matrigel mixed with heparin and bFGF served as a positive control; naive mice injected with Matrigel alone were used as a negative control. Mice were euthanized on day 16. The Matrigel plugs were resected from surrounding connective tissues. Half of the Matrigel plugs were fixed in 10\% formaldehyde, embedded in paraffin, sectioned, and stained with hematoxylin and eosin or Giemsa stains to calculate microvessel density. In each section, the five most vascular areas were chosen. Microvessel counts were obtained at $\times 400$, and the mean number in the five fields for the Matrigel plugs was calculated and referred to as the MVD count. The remaining half of the Matrigel plugs were assayed for hemoglobin content according to manufacturer's instructions (Drabkin's reagent kit; Sigma Diagnostics Co., St. Louis, Missouri, USA). 


\section{Figure 1}

Confocal fluorescent microscopy to demonstrate the expression and distribution of E7 and chimeric CRT/E7 proteins. The $293 \mathrm{D}^{\mathrm{b}}, \mathrm{K}^{\mathrm{b}}$ cells were transfected with pcDNA3-E7/GFP $(\mathbf{a}-\mathbf{c})$ or pcDNA3- CRT/E7/GFP DNA (d-f). Immunofluorescent staining was performed as described in Methods. For the detection of GFP protein, green fluorescence was noted (b and $\mathbf{e}$ ). For the detection of endogenous calnexin protein, red fluorescence was observed (a and d). Controls omitting primary Ab's did not demonstrate specific red fluorescence (data not shown). Colocalization of GFP and calnexin was demonstrated by the yellow color in the combined image ( $\mathbf{c}$ and $\mathbf{f}$ ).
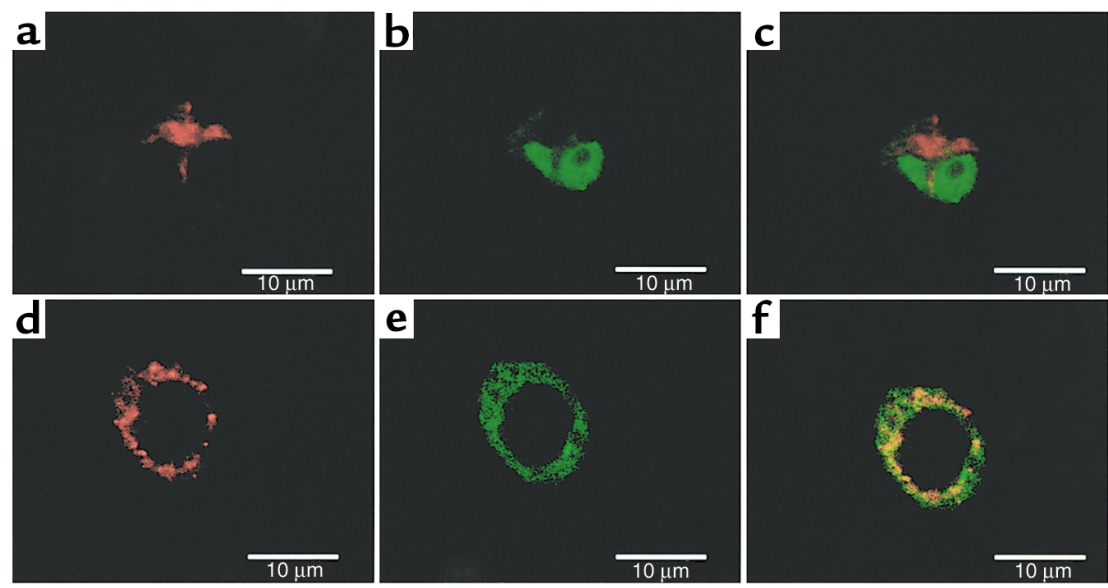

\section{Results}

Confocal fluorescent microscopic examination reveals that the linkage of CRT to E7 targets the antigen to the ER. We have characterized the expression of E7 in the lysates and supernatant from cells transfected with wild-type E7 or CRT/E7 DNA by Western blot analysis (data not shown). Analysis of supernatants from cell cultures revealed that protein was secreted from cells transfected with CRT/E7 DNA but not from E7-transfected cells. Previous studies have demonstrated that CRT may aid in antigen presentation by associating with peptides delivered into the ER by TAP-1 and TAP-2 (8) and with $\mathrm{MHC}$ class I- $\beta 2 \mathrm{~m}$ (9). To determine whether linkage to CRT affected the subcellular localization of E7, we linked the GFP gene to the $3^{\prime}$ end of the E7 gene or the chimeric CRT/E7 gene in the pcDNA3 vector. Transfection and subsequent examination with a confocal fluorescent microscope revealed that cells transfected with E7/GFP DNA showed homogeneous cytoplasmic/nuclear distribution (Figure 1b), while cells transfected with

\section{Figure 2}

Immunological profile of vaccinated mice using intracellular cytokine staining and ELISA. Mice were immunized and splenocytes were prepared as described in Methods. The number of (a) IFN$\gamma$-secreting CD8 ${ }^{+} \mathrm{T}$ cell precursors, (b) IFN- $\gamma$-secreting $\mathrm{CD}^{+}{ }^{+} \mathrm{T}$ cells, and (c) IL-4-secreting CD4 ${ }^{+} \mathrm{T}$ cells in the presence (filled columns) or absence (open columns) of the corresponding E7 peptide (aa 49-57 for CD8, aa 30-67 for CD4) was analyzed using flow cytometry. Data are expressed as mean number of IFN-secreting $T$ cells per $3 \times 10^{5}$ splenocytes \pm SEM. (d) ELISA demonstrates E7-specific $A b$ 's in mice vaccinated with various DNA vaccines. The results from the $1: 100,1: 500$, and 1:1,000 dilution are presented, showing mean absorbance (OD $450 \mathrm{~nm}) \pm$ SEM. The data collected from all of the above experiments are from one representative experiment of two performed.

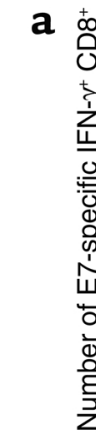

the CRT/E7/GFP DNA construct displayed a network pattern consistent with ER localization (Figure 1e). To confirm that the CRT/E7/GFP chimera had in fact been distributed to the ER, we performed immunofluorescent staining of cells transfected with either E7/GFP or CRT/E7/GFP using an Ab against calnexin (Figure 1, a and d), a well-characterized marker for the ER. As shown in Figure 1, $\mathrm{c}$ and $\mathrm{f}$, colocalization with the calnexin protein was observed in cells transfected with CRT/E7/GFP but not in cells transfected with E7/GFP, indicating that the fusion of CRT to E7/GFP facilitated the entry of the protein into the ER.

Vaccination with CRT/E7 DNA significantly enhances E7-specific $\mathrm{CD}^{+} \mathrm{T}$ cell-mediated immune responses and E7-specific Ab responses. To determine if CRT/E7 DNA
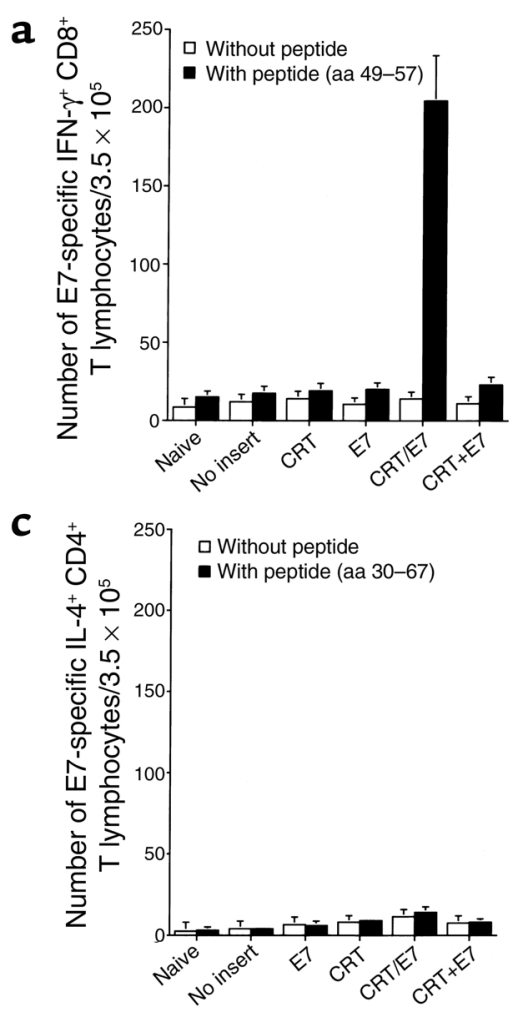

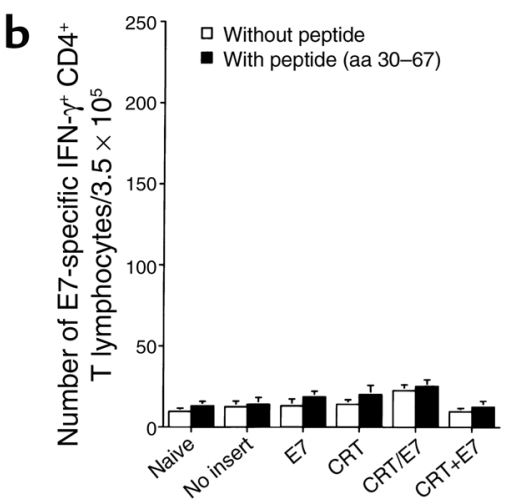

d

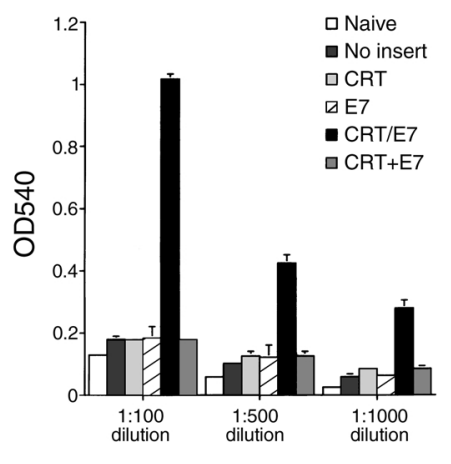




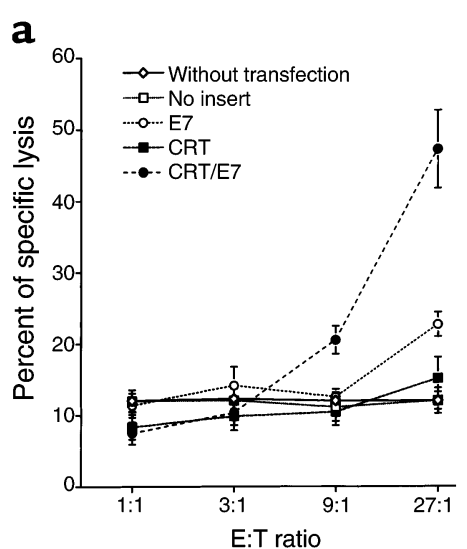

b

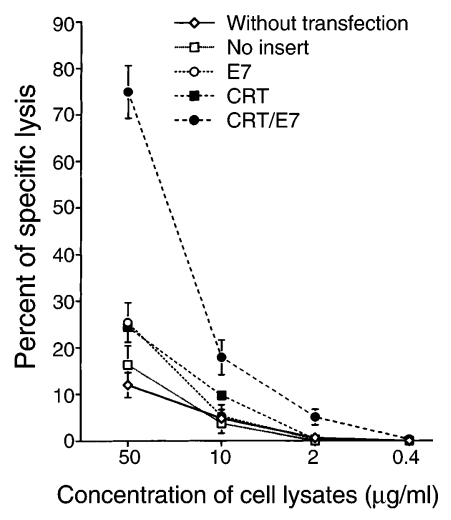

\section{Figure 3}

$C T L$ assays to demonstrate enhanced presentation of E7 through the MHC class I pathway in cells transfected with CRT/E7 DNA and in bone marrow-derived DCs pulsed with cell lysates containing chimeric CRT/E7 protein. (a) CTL assays with various $E / T$ ratios $(E / T=1: 1,3: 1,9: 1,27: 1)$ were performed as described in Methods. The $293 \mathrm{D}^{\mathrm{b}}, \mathrm{K}^{\mathrm{b}}$ cells transfected with various DNA constructs were used as target cells while $\mathrm{D}^{\mathrm{b}}$-restricted E7-specific CD8 ${ }^{+} \mathrm{T}$ cells were used as effector cells. (b) CTL assays were performed at a fixed $E / T$ ratio of 9:1 using bone marrow-derived DCs pulsed with cell lysates from $293 \mathrm{D}^{\mathrm{b}}, \mathrm{K}^{\mathrm{b}}$ cells transfected with various DNA constructs at different concentrations as target cells and $\mathrm{D}^{\mathrm{b}}$-restricted E7-specific $\mathrm{CD} 8^{+}$ $T$ cells as effector cells. The results presented in this figure are from one representative experiment of two performed. could enhance E7-specific $\mathrm{T}$ cell-mediated immune responses in mice, we performed intracellular cytokine staining with flow cytometry analysis to characterize E7-specific $\mathrm{CD}^{+}$and $\mathrm{CD}^{+} \mathrm{T}$ cell precursors. As shown in Figure 2a, vaccination with CRT/E7 DNA generated the highest frequency of E7-specific IFN- $\gamma$-secreting $\mathrm{CD}^{+}$ T cell precursors $\left(204 / 3.5 \times 10^{5}\right.$ splenocytes $)$ compared with mice vaccinated with E7 DNA $\left(18 / 3.5 \times 10^{5}\right.$ splenocytes $)$ $(P<0.01)$. In contrast, DNA encoding E7 fused with irrelevant protein such as GFP did not demonstrate specific enhancement of E7-specific CD8 ${ }^{+} \mathrm{T}$ cell precursors (data not shown). These results also indicated that the fusion of CRT to E7 was required for enhancement of $\mathrm{CD}^{+} \mathrm{T}$ cell activity, since CRT DNA mixed with E7 DNA did not generate enhancement of $\mathrm{CD}^{+} \mathrm{T}$ cell activity. We observed no increase in the number of E7-specific IFN- $\gamma$-secreting or IL-4-secreting $\mathrm{CD}^{+} \mathrm{T}$ cells in mice vaccinated with CRT/E7 DNA compared with mice vaccinated with control plasmid, E7, CRT, or CRT+E7 DNA (Figure 2, b and c). As shown in Figure 2d, vaccination with CRT/E7 DNA generated significantly higher titers of anti-E7 Ab's in the sera of mice compared with the other vaccinated groups $(P<0.01)$.

Enhanced presentation of $E 7$ through the MHC class I pathway in cells transfected with CRT/E7 DNA and in dendritic cells pulsed with chimeric CRT/E7 protein. We explored potential explanations for the observed increase in E7-specific $\mathrm{CD}^{+} \mathrm{T}$ cell precursors in mice vaccinated with CRT/E7 DNA. One potential explanation is that there was direct enhancement of MHC class I presentation of E7 in cells expressing CRT/E7. We therefore performed a CTL assay using $293 \mathrm{D}^{\mathrm{b}}, \mathrm{K}^{\mathrm{b}}$ cells transfected with the various DNA constructs as target cells and an $\mathrm{H}-2$ $\mathrm{D}^{\mathrm{b}}$-restricted E7-specific $\mathrm{CD}^{+} \mathrm{T}$ cell line as effector cells (21). The $293 \mathrm{D}^{\mathrm{b}}, \mathrm{K}^{\mathrm{b}}$ cells were used as target cells since they express the $\mathrm{H}-2 \mathrm{D}^{\mathrm{b}}$ murine MHC class I molecule (28) and have a stable high transfection efficiency (up to 80\%). As shown in Figure 3a, 293 $\mathrm{D}^{\mathrm{b}}, \mathrm{K}^{\mathrm{b}}$ cells transfected with CRT/E7 DNA generated significantly higher percentages of specific lysis at the $9: 1(20.5 \% \pm 1.0 \%$ vs. $10.43 \% \pm 0.9 \%, P<0.001)$ and $27: 1(47.1 \% \pm 5.5 \%$ vs. $15.1 \% \pm 3.0 \%, P<0.001)$ $\mathrm{E} / \mathrm{T}$ ratios compared with mice vaccinated with wildtype E7 DNA. Our results suggested that cells transfected with CRT/E7 DNA were capable of directly presenting E7 antigen through the MHC class I pathway to $\mathrm{CD}^{+} \mathrm{T}$ cells more efficiently than cells transfected with wild-type E7 DNA.

Another potential mechanism for the observed enhancement of E7-specific $\mathrm{CD}^{+} \mathrm{T}$ cell immune responses in vivo is the so-called "cross-priming" effect (29), whereby CRT/E7 protein released from cells may be taken up and processed by other antigenpresenting cells (APCs) via the MHC class I-restricted pathway. We performed CTL assays to characterize MHC class I presentation of E7 in DCs that have taken up exogenous protein. This was modeled by
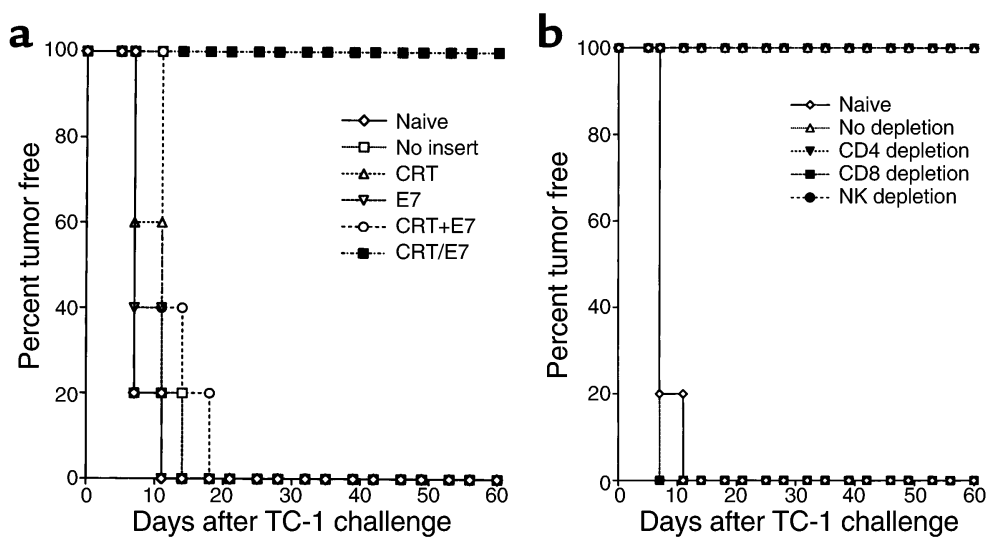

\section{Figure 4}

In vivo tumor protection experiments in mice vaccinated with various DNA vaccines and in vivo Ab depletion experiments in mice vaccinated with CRT/E7. (a) Mice were immunized with various DNA vaccines and challenged as described in the Methods to assess the antitumor effect generated by each DNA vaccine. (b) Mice were immunized with CRT/E7 DNA and challenged with TC-1 tumor cells to determine the effect of lymphocyte subsets on the potency of the CRT/E7 DNA vaccine. CD4, CD8, and NK1.1 depletions were initiated 1 week before tumor challenge and lasted 40 days after tumor challenge. The data from the $A b$ depletion experiments shown here are from one representative experiment of two performed. 

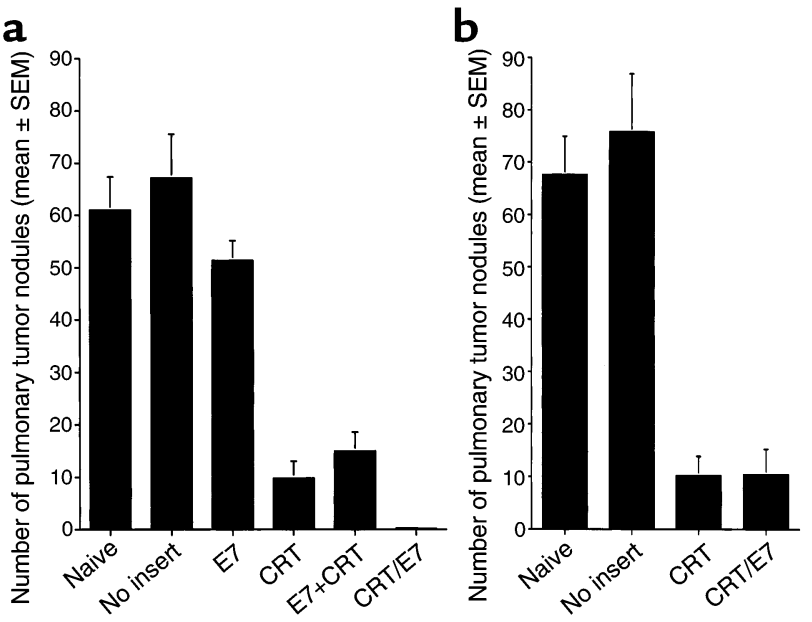

\section{Figure 5}

In vivo tumor treatment experiments in C57BL/ 6 mice and C57BL/ 6 mice depleted of both $\mathrm{CD} 4^{+}$and $\mathrm{CD} 8^{+} \mathrm{T}$ cells that are treated with various DNA constructs. Mice were challenged and subsequently treated with various DNA vaccines at a high therapeutic dose as described in Methods. (a) Tumor treatment experiments in C57BL/ 6 mice. (b) Tumor treatment experiments in $\mathrm{C} 57 \mathrm{BL} / 6$ mice depleted of both $\mathrm{CD}^{+}$and $\mathrm{CD} 8^{+} \mathrm{T}$ cells. The data are expressed as mean number of pulmonary lung nodules \pm SEM. Data shown are from one representative experiment of two performed.

pulsing DCs with cell lysates of $293 \mathrm{D}^{\mathrm{b}}, \mathrm{K}^{\mathrm{b}}$ cells transfected with the various DNA constructs. As shown in Figure 3b, DCs pulsed with lysates of $293 \mathrm{D}^{\mathrm{b}}, \mathrm{K}^{\mathrm{b}}$ cells transfected with CRT/E7 DNA generated a significantly higher percentage of specific lysis than naive DCs or DCs pulsed with lysates of $293 \mathrm{D}^{\mathrm{b}}, \mathrm{K}^{\mathrm{b}}$ cells transfected with other DNA constructs $(P<0.001)$. These results indicated that the linkage of CRT to E7 may enhance MHC class I presentation of E7 by DCs when antigen is administered exogenously.

Vaccination with CRT/E7 DNA enhances tumor protection in mice challenged with an E7-expressing tumor cell line. To determine if the observed enhancement in E7-specific $\mathrm{CD}^{+} \mathrm{T}$ cell-mediated immunity translated to a significant E7-specific antitumor effect, we performed an in vivo tumor protection experiment using a previously characterized E7-expressing tumor model, TC-1 (22). As shown in Figure $4 a, 100 \%$ of mice receiving CRT/E7 DNA vaccination remained tumor-free 60 days after TC- 1 challenge. In contrast, all of the unvaccinated mice and mice receiving plasmid without insert, CRT, or E7 DNA developed tumors within 15 days after tumor challenge. Furthermore, mice vaccinated with DNA encoding E7 fused with irrelevant protein such as GFP did not show a significant antitumor effect (data not shown). Our results also indicated that the fusion of CRT to E7 was required for antitumor immunity, since CRT mixed with E7 (CRT + E7 DNA) did not generate significant enhancement of antitumor immunity.

$C D 8^{+} T$ cells but not $C D 4^{+} T$ cells or natural killer cells are essential for the antitumor effect generated by CRT/E7 DNA. To determine the subset of lymphocytes important for the antitumor effect, we performed in vivo Ab depletion experiments (22). As shown in Figure 4b, all naive mice and all mice depleted of $\mathrm{CD}^{+} \mathrm{T}$ cells grew tumors within 15 days after tumor challenge. In contrast, all of the nondepleted mice and all of the mice depleted of $\mathrm{CD}^{+} \mathrm{T}$ cells or NK1.1 cells remained tumor-free 60 days after tumor challenge. These results suggested that $\mathrm{CD}^{+} \mathrm{T}$ cells are important for the antitumor immunity generated by the CRT/E7 DNA vaccine.

Treatment with CRT/E7 leads to significant reduction of pulmonary tumor nodules in C57BL/6 mice. The therapeutic potential of each vaccine was assessed by performing an in vivo tumor treatment experiment using a previously described lung hematogenous spread model (23). As shown in Figure 5a, C57BL/6 mice treated with CRT/E7 DNA exhibited the lowest mean number of pulmonary tumor nodules $(0.2 \pm 0.4)$, significantly lower than mice treated with CRT DNA $(9.8 \pm 2.3)$ or wild-type E7 DNA $(51.4 \pm 3.7)$ (one-way ANOVA, $P<0.001)$. These data indicated that CRT/E7 DNA generated the most potent antitumor effect compared with CRT or wild-type E7 DNA constructs in the lung hematogenous spread model. We also found that fusion of CRT to E7 was important to the antitumor effect since vaccination with CRT mixed with E7 (CRT + E7) did not generate as robust an effect $(15.0 \pm 2.6$ tumor nodules) as vaccination with CRT/E7 (0.2 \pm 0.4 tumor nodules). Interestingly, treatment with wild-type CRT DNA also resulted in significantly fewer tumor nodules than treatment with wild-type E7 DNA or no treatment (one-way ANOVA, $P<0.001$ ).

Reduction of pulmonary tumor nodules in CRT or CRT/E7 DNA-treated C57BL/6 mice that are depleted of both $C D 4^{+}$ and $C D 8^{+} T$ cells. We observed that CRT alone generated a therapeutic effect against TC-1 tumors (Figure 5a). Since wild-type CRT does not contain E7, we reasoned that the therapeutic effect of wild-type CRT was likely not related to $\mathrm{T}$ cell-mediated immune responses, but rather was mediated by $\mathrm{T}$ cell-independent mechanisms such as antiangiogenesis. To evaluate the role of CRT/E7 and CRT DNA in the treatment of TC-1 tumors in the lungs without the involvement of $\mathrm{T}$ cells, we used C57BL/ 6 mice depleted of both $\mathrm{CD}^{+}$and $\mathrm{CD}^{+} \mathrm{T}$ cells for in vivo tumor treatment experiments. Our results revealed that double-depleted mice treated with CRT/E7 DNA or CRT DNA exhibited significantly fewer pulmonary tumor nodules $(10.2 \pm 3.7$ for CRT, $10.4 \pm 4.9$ for CRT/E7) than naive mice $(67.6 \pm 7.3)$, or mice receiving pcDNA3 without insert $(75.8 \pm 11.1)$ (Figure $5 b$ ). There was no significant difference in the number of pulmonary nodules in double-depleted mice treated with either CRT/E7 DNA or CRT DNA. Taken together, these data indicate that CRT and CRT/E7 DNA are able to generate an antitumor effect independent of $\mathrm{T}$ cell-mediated immune responses. We reasoned that the therapeutic effect observed with CRTcontaining vaccines may be related to antiangiogenesis.

Reduction of pulmonary tumor nodules in nude mice challenged with TC-1 and treated with CRT or chimeric CRT/E7 

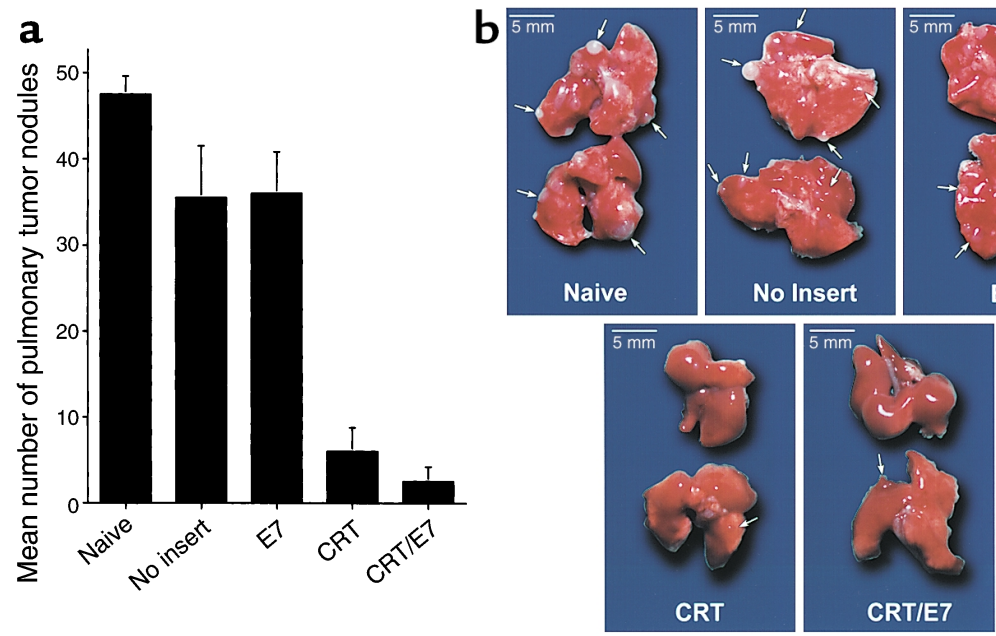

Figure 6

In vivo tumor treatment experiments to compare the antitumor effect generated by CRT and CRT/E7 DNA vaccines in nude mice. (a) Tumor treatment experiments in nude (BALB/c $n u / n u)$ mice. Mice were intravenously challenged with TC-1 tumor cells and treated with various DNA vaccines as described in Methods. The mean number of pulmonary tumor nodules ( \pm SEM) shown here is from one representative experiment of two performed. (b) Representative gross pictures of pulmonary tumors in each vaccinated group.

DNA vaccines. To further confirm that treatment with CRT or CRT/E7 DNA could generate a T cell-independent anti-tumor effect, we performed an in vivo tumor treatment experiment using immunocompromised (BALB/c nu/nu) mice. As shown in Figure 6a, nude mice treated with CRT or CRT/E7 DNA displayed a significantly lower mean number of pulmonary tumor nodules $(6.0 \pm 2.8$ for CRT, $2.5 \pm 0.7$ for CRT/E7) compared with mice treated with wildtype E7 DNA $(36.0 \pm 2.8)$, plasmid without insert $(35.5 \pm 12.0)$, or naive group $(47.5 \pm 2.1)$ (one-way ANOVA, $P<0.001)$. Interestingly, nude mice treated with CRT/E7 DNA exhibited a slightly greater antitumor effect than nude mice treated with CRT DNA. Representative gross photographs of the pulmonary tumors are shown in Figure 6b. These data support the results in Figure 5b that CRT and CRT/E7 DNA

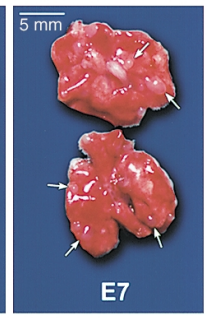

are able to generate an antitumor effect even in the absence of $\mathrm{T}$ cell-mediated immune responses.

Reduction in microvessel density of tumors in nude mice challenged with TC-1 and treated with CRT or chimeric CRT/E7 DNA vaccines. To determine whether the antitumor effect of CRT and CRT/E7 DNA in the absence of $T$ cells might be mediated through antiangiogenesis, we measured MVD in the pulmonary tumors of nude mice treated with various DNA vaccines. As shown in Figure $7 \mathrm{a}$, treatment of nude mice with either CRT or CRT/E7 DNA resulted in significantly lower MVD in pulmonary tumors than treatment with wild-type E7 or plasmid without insert (one-way ANOVA, $P<0.001)$. MVD in pulmonary tumors was slightly lower in nude mice treated with CRT/E7 DNA than in nude mice treated with CRT DNA. Representative photographs of immunohistochemical labeling of sections from lung nodules obtained from different vaccinated nude mice are shown in Figure $7 \mathrm{~b}$.

In vivo antiangiogenesis in mice treated with CRT or CRT/E7 $D N A$. To provide a more quantitative assessment of antiangiogenesis in C57BL/6 mice treated with the various DNA constructs, we performed an in vivo angiogenesis assay using a Matrigel assay (26). As shown in Figure 8a, the hemoglobin content of Matrigel implants from CRT and CRT/E7 DNA-treated mice was significantly lower than it was in control and E7 DNA-treated mice $(P<0.01$, ANOVA). There was no statistically significant difference in hemoglobin content between CRT and CRT/E7 Matrigel samples. This assay revealed that CRT or CRT/E7 DNA could generate a similar degree of inhibition of bFGF-induced in vivo angiogenesis. We also examined the MVD of Matrigel samples to provide
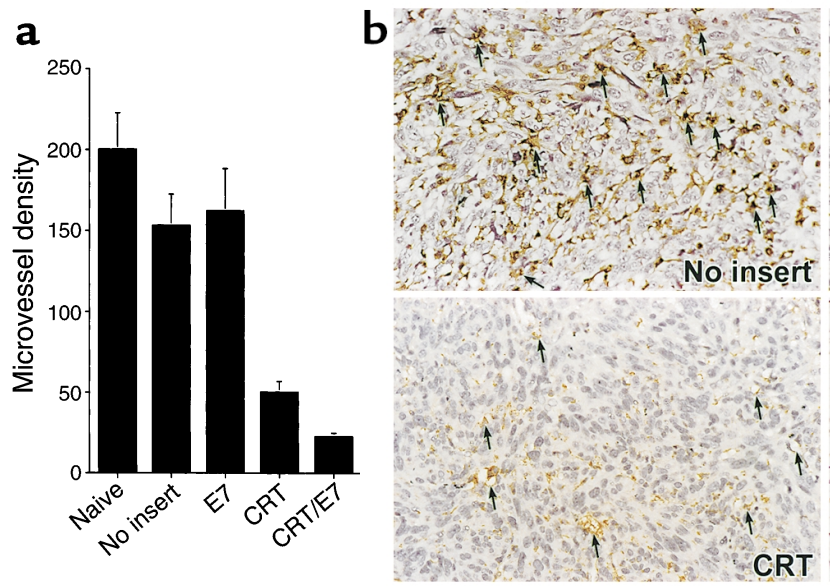

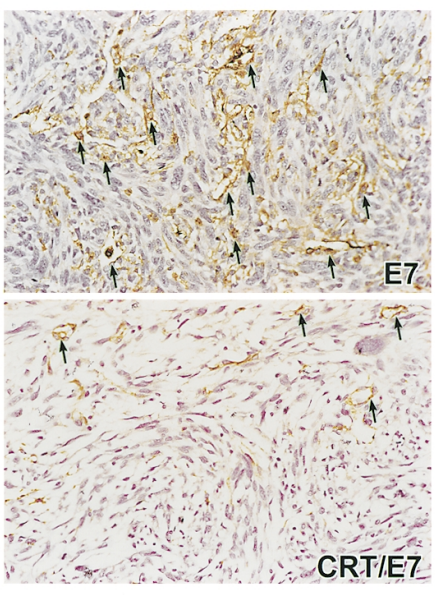

\section{Figure 7}

Determination of the MVD of pulmonary tumor nodules in DNA-treated nude mice. Immunohistochemical labeling and microvessel counts were performed as described in Methods. (a) The mean MVD is shown for each of the vaccination groups. (b) Representative photographs of immunohistochemical labeling in sections from lung nodules obtained from different vaccinated nude mice. Positive labeling of intratumoral microvessels is indicated with arrows. 


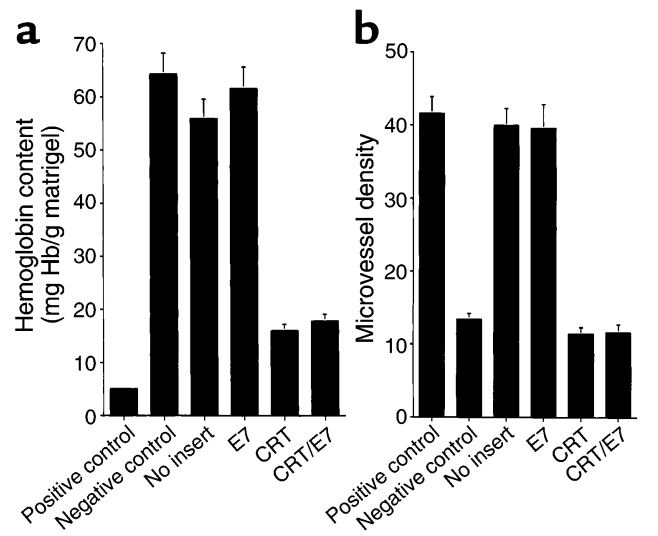

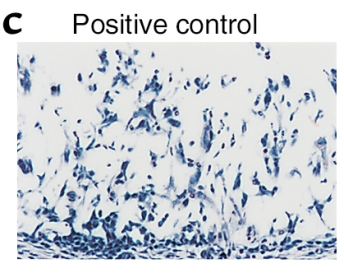

E7

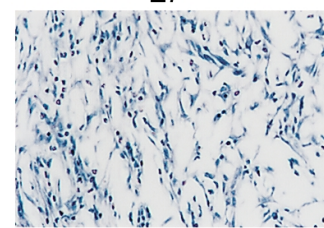

Negative control

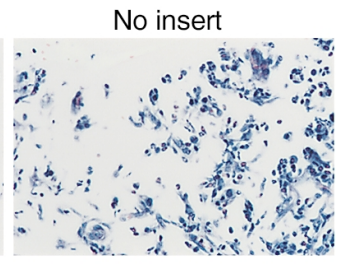

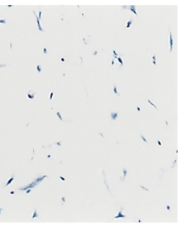

CRT
CRT/E7

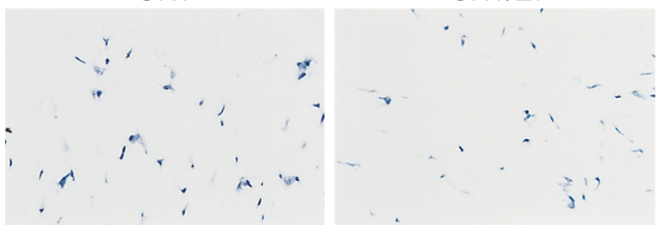

Figure 8

In vivo angiogenesis assay using Matrigel to characterize the antiangiogenic effect mediated by treatment with various DNA constructs. Mice were treated with various DNA constructs and injected with Matrigel as described in Methods. Nine days later, mice were euthanized, and Matrigel plugs were resected. (a) Matrigel hemoglobin content using the Drabkin method. The mean hemoglobin content ( \pm SEM) shown here is from one representative experiment of two performed. (b) Mean MVD in Matrigel samples from each vaccination group. (c) Representative photographs of sections of Matrigel samples from each vaccination group (Giemsa stain, $\times 400$ ).

another measure of angiogenesis inhibition since red blood cells may extravasate from vessels and affect the hemoglobin count. The mean MVD in Matrigel samples from CRT (11.4 \pm 0.9$)$ and CRT/E7 (11.6 \pm 1.1$)$ DNAtreated mice was similar and significantly lower than the MVD in Matrigel samples from pcDNA3 without insert $(40.0 \pm 2.3)$ or wild-type E7 $(39.6 \pm 3.3)$ DNA-treated mice (Figure 8b). Representative sections of Matrigel samples after Giemsa staining are shown in Figure 8c. We also determined the serum concentration of CRT or CRT/E7 protein in mice treated with CRT or CRT/E7 DNA. We found that the peak serum concentration of both CRT and CRT/E7 protein was approximately 15 $\mathrm{ng} / \mathrm{ml}$ on day 11 after the last vaccination, while CRT protein was undetectable in mice without vaccination (data not shown). Taken together, these results demonstrate that treatment of mice with CRT or CRT/E7 DNA can lead to systemic release of CRT or CRT/E7 protein and inhibition of angiogenesis.

\section{Discussion}

In this study, we demonstrated that linkage of HPV-16 E7 antigen to CRT can significantly enhance the potency of an E7-expressing DNA vaccine. CRT/E7 DNA elicited strong $\mathrm{E} 7$-specific $\mathrm{CD}^{+} \mathrm{T}$ cell immune responses, generated a significant $\mathrm{CD}^{+} \mathrm{T}$ cell-dependent protective effect against subcutaneous HPV-16 E7-expressing tumors, and was useful in the treatment of lethal pulmonary tumor nodules. Furthermore, the CRT/E7 DNA vaccine also generated a therapeutic effect due to inhibition of angiogenesis, which likely contributed to reduction of pulmonary tumor nodules. Thus, DNA vaccines encoding CRT chimerically linked to a tumor antigen represent a unique approach that combines immunological and antiangiogenic approaches for the generation of a potent antitumor effect.

Our data demonstrated that mice vaccinated with CRT/E7 DNA exhibited enhanced E7-specific CD8 ${ }^{+}$
$\mathrm{T}$ cell responses. One of the possible mechanisms for this enhancement is the chaperone effect of CRT, which may enhance MHC class I presentation of E7. Several studies have indicated that ER targeting may be capable of enhancing antigen-specific MHC class I-restricted CTL activity (30-33). Since ballistic DNA delivery can introduce DNA directly into dermal professional APCs, CRT/E7 DNA-transfected APCs may directly enhance the presentation of E7 through the MHC class I pathway to $\mathrm{CD}^{+} \mathrm{T}$ cells and contribute to the generation of E7-specific $\mathrm{CD} 8^{+} \mathrm{T}$ cell precursors in vivo. Another mechanism that may play a role in the enhancement of E7-specific $\mathrm{CD}^{+} \mathrm{T}$ cell immune responses in vivo is the so-called "cross-priming" effect, whereby secretion of CRT/E7 protein or lysis of cells expressing CRT/E7 antigen releases the chimeric protein exogenously to be taken up and processed by other APCs via the MHC class I-restricted pathway (29, 34-37). One recent study found that CD91, an $\alpha 2$ macroglobulin receptor, serves as a receptor for heat shock proteins (HSPs), including CRT, gp96, HSP70, and HSP90 (38). Peptides that are complexed with these HSPs can be taken up by macrophages and dendritic cells and presented on MHC class I molecules. These mechanisms may provide an explanation for the observed enhancement of E7-specific $\mathrm{CD}^{+} \mathrm{T}$ cell activity in mice vaccinated with CRT/E7 DNA.

We observed that E7-specific Ab titers were significantly enhanced by CRT/E7 vaccination, despite lack of enhancement of E7-specific $\mathrm{CD}^{+} \mathrm{T}$ helper cells. Although the mechanism for enhancement of $\mathrm{Ab}$ responses is not clear, we cannot exclude the possibility that there were $\mathrm{T}$ helper cells generated against CRT. Even though Ab-mediated responses have not been shown to play an important role in controlling HPVassociated malignancies, antigen-specific $\mathrm{Ab}$ 's are significant in other tumor models such as the breast cancer model with the HER-2/neu antigen. The chimeric CRT 
vaccine strategy may be used to generate HER-2/neu-specific Ab's. HER-2/neu-specific Ab's have been shown to induce growth arrest in cells expressing high levels of HER-2/neu on the cell surface (39).

We observed that treatment of CD4/CD8 doubledepleted C57BL/ 6 mice with CRT/E7 or CRT DNA generated comparable $\mathrm{T}$ cell-independent antitumor effects. Meanwhile, tumor treatment experiments and examination of microvessel density in nude mice revealed that CRT/E7 DNA generated a stronger effect than CRT DNA. This observation may be related to the different genetic backgrounds of the mice (C57BL/6 vs. BALB/c nu/nu). For example, the production and effects of cytokines or chemokines may vary in mice of different genetic backgrounds, which is significant since these molecules have been shown to affect tumor growth and angiogenesis (for review, see refs. 40, 41). Also, there may be differences in the activity of non-T cell effectors (i.e., natural killer cells, neutrophils, and macrophages) in these mice; such effector cells may affect the antitumor effect and angiogenesis. Variations in these characteristics may contribute to the differences observed in $\mathrm{C} 57 \mathrm{BL} / 6$ mice depleted of both $\mathrm{CD}^{+}$and $\mathrm{CD}^{+} \mathrm{T}$ cells and $\mathrm{BALB} / \mathrm{c} n u / n u$ mice treated with the CRT/E7 or CRT DNA vaccines. It would be interesting in the future to investigate characteristics of cytokine production and non-T cell effectors related to vaccination with CRT/E7 DNA versus CRT DNA and examine their role in the antitumor effect and antiangiogenesis.

The encouraging results from this study suggest that gene therapy using naked DNA expressing other inhibitors of angiogenesis may also lead to a therapeutic antitumor effect. Several agents that target tumor vasculature have been shown to prevent or delay tumor growth and even promote tumor regression or dormancy. These agents may thus be useful in gene therapy applications. For example, angiostatin, a fragment of plasminogen (42), and endostatin, a fragment of collagen XVIII (43), have been shown to suppress neovascularization and inhibit the growth of a variety of experimental tumors. IFN- $\gamma(44)$, the IFN- $\gamma$ inducible protein-10 (45), IL-12 $(27,46,47)$, a fragment of prolactin (48), thrombospondin (49), and platelet factor-4 (50) are examples of various other compounds that have been shown to inhibit angiogenesis and exert an antitumor effect. These molecules potentially can be used for the development of DNA vaccines for the control of tumors. In addition, DNA molecules may be easier to prepare and deliver than protein molecules and administration of DNA expressing these antiangiogenic agents may lead to persistent expression and release of these therapeutic proteins over a prolonged period of time, resulting in control of tumor growth. Thus, there is considerable enthusiasm for the application of DNA expressing inhibitors of angiogenesis for cancer treatment.

While the CRT/E7 DNA vaccine may hold promise for mass immunization, certain safety issues need to be resolved. There exists the concern that DNA may integrate into the host genome, although it is estimated that the frequency of such integration is much lower than that of spontaneous mutation and should not pose a significant risk (51). Another issue is related to HPV-16 E7 protein, which is known to disrupt cell cycle regulation by binding to the tumor suppressor $\mathrm{PRB}$ in nuclei (52). The oncogenicity of E7 can be eliminated by using mutated E7 that is unable to bind with $\mathrm{pRB}$ (53) but still maintains most of its antigenicity. Finally, there may be concerns about the generation of autoimmunity related to CRT (for review, see ref. 54). In our study, we performed pathological examination of the vital organs in CRT/E7-vaccinated mice. We did not observe abnormal inflammation or pathology (data not shown), indicating that CRT/E7 is a potent vaccine with minimal malevolent side effects.

In summary, our results indicate that fusion of CRT to HPV-16 E7 gene can generate an impressive antitumor effect against HPV-16 E7-expressing murine tumors through enhancement of E7-specific $\mathrm{CD}^{+} \mathrm{T}$ cell-mediated immune responses and antiangiogenesis. The fusion of CRT to an antigen gene is a promising approach to cancer therapy that potentially can be applied to other cancer systems with known tumor-specific antigens.

\section{Acknowledgments}

We thank Drew M. Pardoll, Robert J. Kurman, Yvonne Paterson, and Chang-Yao Hsieh for helpful discussions. CRT cDNA was kindly provided by Marek Michalak (University of Alberta, Edmonton, Alberta, Canada). We would also like to thank James C. Yang (National Cancer Institute, NIH, Bethesda, Maryland, USA) for kindly providing $293 \mathrm{D}^{\mathrm{b}}, \mathrm{K}^{\mathrm{b}}$ cells and Ralph Hruban, Ken-Yu Lin, and Richard Roden for critical review of the manuscript. This work was supported by the National Cancer Institute, the Cancer Research Institutes, and the Alexander and Margaret Stewart Trust grant. Dr. Wen-Fang Cheng is a recipient of the NHRI physician scientist fellowship (RE88P005).

1. Boon, T., Cerottini, J.C., Van den Eynde, B., van der Bruggen, P., and Van Pel, A. 1994. Tumor antigens recognized by T lymphocytes. Annu. Rev. Immunol. 12:337-365.

2. Chen, C.H., and Wu, T.C. 1998. Experimental vaccine strategies for cancer immunotherapy. J. Biomed. Sci. 5:231-252.

3. Folkman, J. 1982. Angiogenesis: initiation and control. Ann. NY Acad. Sci. 401:212-227.

4. Hanahan, D., and Folkman, J. 1996. Patterns and emerging mechanisms of the angiogenic switch during tumorigenesis. Cell. 86:353-364.

5. Nash, P.D., Opas, M., and Michalak, M. 1994. Calreticulin: not just another calcium-binding protein. Mol. Cell. Biochem. 135:71-78.

6. Conway, E.M., et al. 1995. Heat shock-sensitive expression of calreticulin. In vitro and in vivo up-regulation. J. Biol. Chem. 270:17011-17016.

7. Basu, S., and Srivastava, P.K. 1999. Calreticulin, a peptide-binding chaperone of the endoplasmic reticulum, elicits tumor- and peptide-specific immunity. J. Exp. Med. 189:797-802.

8. Spee, P., and Neefjes, J. 1997. TAP-translocated peptides specifically bind proteins in the endoplasmic reticulum, including gp 96 , protein disulfide isomerase and calreticulin. Eur. J. Immunol. 27:2441-2449.

9. Sadasivan, B., Lehner, P.J., Ortmann, B., Spies, T., and Cresswell, P. 1996. Roles for calreticulin and a novel glycoprotein, tapasin, in the interaction of MHC class I molecules with TAP. Immunity. 5:103-114.

10. Folkman, J. 1995. Angiogenesis in cancer, vascular, rheumatoid and other disease. Nat. Med. 1:27-31. 
11. Pike, S.E., et al. 1999. Calreticulin and calreticulin fragments are endothelial cell inhibitors that suppress tumor growth. Blood. 94:2461-2468

12. Pike, S.E., et al. 1998. Vasostatin, a calreticulin fragment, inhibits angiogenesis and suppresses tumor growth. J. Exp. Med. 188:2349-2356.

13. Hung, C.F., et al. 2001. Cancer immunotherapy using a DNA vaccine encoding the translocation domain of a bacterial toxin linked to a tumor antigen. Cancer Res. 61:3698-3703.

14. Michalak, M., Corbett, E.F., Mesaeli, N., Nakamura, K., and Opas, M. 1999. Calreticulin: one protein, one gene, many functions. Biochem. J. 344:281-292.

15. Hung, C.F., et al. 2001. Enhancement of DNA vaccine potency by linkage of antigen gene to a gene encoding the extracellular domain of Fmslike tyrosine kinase 3-ligand. Cancer Res. 61:1080-1088.

16. Chen, C.H., et al. 2000. Enhancement of DNA vaccine potency by linkage of antigen gene to an HSP70 gene. Cancer Res. 60:1035-1042.

17. Feltkamp, M.C., et al. 1993. Vaccination with cytotoxic T lymphocyte epitope-containing peptide protects against a tumor induced by human papillomavirus type 16-transformed cells. Eur. J. Immunol. 23:2242-2249.

18. Tindle, R.W., Fernando, G.J., Sterling, J.C., and Frazer, I.H. 1991. A "public" T-helper epitope of the E7 transforming protein of human papillomavirus 16 provides cognate help for several E7 B-cell epitopes from cervical cancer-associated human papillomavirus genotypes. Proc. Natl. Acad. Sci. USA. 88:5887-5891.

19. Cheng, W.F., et al. 2001. Enhancement of sindbis virus self-replicating RNA vaccine potency by linkage of herpes simplex virus type 1 VP22 protein to antigen. J. Virol. 75:2368-2376.

20. Cheng, W.F., et al. 2001. Enhancement of sindbis virus self-replicating RNA vaccine potency by targeting antigen to endosomal/lysosomal compartments. Hum. Gene Ther. 12:235-252.

21. Wang, T.L., et al. 2000. Intramuscular administration of E7-transfected dendritic cells generates the most potent E7-specific anti-tumor immunity. Gene Ther. 7:726-733.

22. Lin, K.-Y., et al. 1996. Treatment of established tumors with a novel vaccine that enhances major histocompatibility class II presentation of tumor antigen. Cancer Res. 56:21-26.

23. Ji, H., et al. 1998. Antigen-specific immunotherapy for murine lung metastatic tumors expressing human papillomavirus type 16 E7 oncoprotein. Int. J. Cancer. 78:41-45.

24. Huang, C.C., et al. 1999. HPV in situ hybridization with catalyzed signal amplification and polymerase chain reaction in establishing cerebellar metastasis of a cervical carcinoma. Hum. Pathol. 30:587-591.

25. Cheng, W.F., et al. 1999. Vascularity index as a novel parameter for the in vivo assessment of angiogenesis in patients with cervical carcinoma. Cancer. 85:651-657.

26. Passaniti, A., et al. 1992. A simple, quantitative method for assessing angiogenesis and antiangiogenic agents using reconstituted basement membrane, heparin, and fibroblast growth factor. Lab Invest. 67:519-528.

27. Coughlin, C.M., et al. 1998. Interleukin-12 and interleukin-18 synergistically induce murine tumor regression which involves inhibition of angiogenesis. J. Clin. Invest. 101:1441-1452.

28. Bloom, M.B., et al. 1997. Identification of tyrosinase-related protein 2 as a tumor rejection antigen for the B16 melanoma. J. Exp. Med. 185:453-459.

29. Huang, A.Y., et al. 1994. Role of bone marrow-derived cells in presenting MHC class I-restricted tumor antigens. Science. 264:961-965.

30. Restifo, N.P., et al. 1995. Antigen processing in vivo and the elicitation of primary CTL responses. J. Immunol. 154:4414-4422.

31. Fu, T.M., et al. 1998. An endoplasmic reticulum-targeting signal sequence enhances the immunogenicity of an immunorecessive simian virus 40 large $\mathrm{T}$ antigen cytotoxic T-lymphocyte epitope. J. Virol. 72:1469-1481.

32. Boyle, J.S., Brady, J.L., and Lew, A.M. 1998. Enhanced responses to a DNA vaccine encoding a fusion antigen that is directed to sites of immune induction. Nature. 392:408-411.

33. Rice, J., King, C.A., Spellerberg, M.B., Fairweather, N., and Stevenson, F.K 1999. Manipulation of pathogen-derived genes to influence antigen presentation via DNA vaccines. Vaccine. 17:3030-3038.

34. Srivastava, P.K., Udono, H., Blachere, N.E., and Li, Z. 1994. Heat shock proteins transfer peptides during antigen processing and CTL priming. Immunogenetics. 39:93-98.

35. Arnold, D., Faath, S., Rammensee, H., and Schild, H. 1995. Cross-priming of minor histocompatibility antigen-specific cytotoxic $\mathrm{T}$ cells upon immunization with the heat shock protein gp96. J. Exp. Med. 182:885-889.

36. Suto, R., and Srivastava, P.K. 1995. A mechanism for the specific immunogenicity of heat shock protein- chaperoned peptides. Science. 269:1585-1588

37. Castellino, F., et al. 2000. Receptor-mediated uptake of antigen/heat shock protein complexes results in major histocompatibility complex class I antigen presentation via two distinct processing pathways. J. Exp. Med. 191:1957-1964.

38. Basu, S., Binder, R.J., Ramalingam, T., and Srivastava, P.K. 2001. CD91 is a common receptor for heat shock proteins gp96, hsp90, hsp70, and calreticulin. Immunity. 14:303-313.

39. Harwerth, I.M., Wels, W., Schlegel, J., Muller, M., and Hynes, N.E. 1993. Monoclonal antibodies directed to the erbB-2 receptor inhibit in vivo tumour cell growth. Br. J. Cancer. 68:1140-1145.

40. Leek, R.D., Harris, A.L., and Lewis, C.E. 1994. Cytokine networks in solid human tumors: regulation of angiogenesis. J. Leukoc. Biol. 56:423-435.

41. Rossi, D., and Zlotnik, A. 2000. The biology of chemokines and their receptors. Annu. Rev. Immunol. 18:217-242.

42. O'Reilly, M.S., et al. 1994. Angiostatin: a novel angiogenesis inhibitor that mediates the suppression of metastases by a Lewis lung carcinoma. Cell. 79:315-328.

43. O'Reilly, M.S., et al. 1997. Endostatin: an endogenous inhibitor of angiogenesis and tumor growth. Cell. 88:277-285.

44. Fathallah-Shaykh, H.M., Zhao, L.J., Kafrouni, A.I., Smith, G.M., and Forman, J. 2000. Gene transfer of IFN-gamma into established brain tumors represses growth by antiangiogenesis. J. Immunol. 164:217-222.

45. Angiolillo, A.L., et al. 1995. Human interferon-inducible protein 10 is a potent inhibitor of angiogenesis in vivo. J. Exp. Med. 182:155-162.

46. Voest, E.E., et al. 1995. Inhibition of angiogenesis in vivo by interleukin 12. J. Natl. Cancer Inst. 87:581-586.

47. Duda, D.G., et al. 2000. Direct in vitro evidence and in vivo analysis of the antiangiogenesis effects of interleukin 12. Cancer Res. 60:1111-1116.

48. Clapp, C., Martial, J.A., Guzman, R.C., Rentier-Delure, F., and Weiner, R.I. 1993. The 16-kilodalton N-terminal fragment of human prolactin is a potent inhibitor of angiogenesis. Endocrinology. 133:1292-1299.

49. Good, D.J., et al. 1990. A tumor suppressor-dependent inhibitor of angiogenesis is immunologically and functionally indistinguishable from a fragment of thrombospondin. Proc. Natl. Acad. Sci. USA. 87:6624-6628

50. Maione, T.E., et al. 1990. Inhibition of angiogenesis by recombinant human platelet factor-4 and related peptides. Science. 247:77-79.

51. Nichols, W.W., Ledwith, B.J., Manam, S.V., and Troilo, P.J. 1995. Potential DNA vaccine integration into host cell genome. Ann. NY Acad. Sci. 772:30-39.

52. Lukas, J., et al. 1994. DNA tumor virus oncoproteins and retinoblastoma gene mutations share the ability to relieve the cell's requirement for cyclin D1 function in G1. J. Cell Biol. 125:625-638.

53. Heck, D.V., Yee, C.L., Howley, P.M., and Munger, K. 1992. Efficiency of binding the retinoblastoma protein correlates with the transforming capacity of the E7 oncoproteins of the human papillomaviruses. Proc. Natl. Acad. Sci. USA. 89:4442-4446.

54. Eggleton, P., and Llewellyn, D.H. 1999. Pathophysiological roles of calreticulin in autoimmune disease. Scand. J. Immunol. 49:466-473. 